\title{
BMJ Open Health promotion at the workplace setting: a protocol for a systematic review of effectiveness and sustainability of current practice in low- income and middle-income countries
}

\author{
Mary Njeri Wanjau, ${ }^{\circledR 1,2}$ Belen Zapata-Diomedi, ${ }^{2,3}$ Lennert Veerman $^{\odot 4}$
}

To cite: Wanjau MN, ZapataDiomedi B, Veerman L. Health promotion at the workplace setting: a protocol for a systematic review of effectiveness and sustainability of current practice in lowincome and middle-income countries. BMJ Open 2019;9:e027050. doi:10.1136/ bmjopen-2018-027050

- Prepublication history and additional material for this paper are available online. To view these files, please visit the journal online (http://dx.doi. org/10.1136/bmjopen-2018027050).

Received 4 October 2018 Revised 16 April 2019 Accepted 17 April 2019

Check for updates

(C) Author(s) (or their employer(s)) 2019. Re-use permitted under CC BY-NC. No commercial re-use. See rights and permissions. Published by BMJ.

${ }^{1}$ School of Nursing, The University of Nairobi, Nairobi, Nairobi, Kenya

${ }^{2}$ School of Medicine, Griffith University, Gold Coast, Queensland, Australia ${ }^{3}$ Centre for Urban Research, RMIT University, Melbourne, Victoria, Australia

${ }^{4}$ School of Medicine, Griffith University, Southport, Queensland, Australia

Correspondence to Miss Mary Njeri Wanjau; mary.wanjau@griffithuni.edu.au

\section{ABSTRACT}

Introduction Low-income and middle-income countries (LMICS) are experiencing a growing disease burden due to non-communicable diseases (NCDs). Changing behavioural practices, such as diets high in saturated fat, salt and sugar and sedentary lifestyles, have been associated with the increase in NCDs. Health promotion at the workplace setting is considered effective in the fight against NCDs and has been reported to yield numerous benefits. However, there is a need to generate evidence on the effectiveness and sustainability of workplace health promotion practice specific to LMICs. We aim to synthesise the current literature on workplace health promotion in LMICs focusing on interventions effectiveness and sustainability.

Methods and analysis We will conduct a systematic review of published studies from LMICs up to 31 March 2019. We will search the following databases: EMBASE, MEDLINE, PubMed, Web of Science, Scopus, ProQuest and CINAHL. Two reviewers will independently screen potential articles for inclusion and disagreements will be resolved by consensus. We will appraise the quality and risk of bias of included studies using two tools from the Cochrane handbook for systematic reviews of interventions. We will present a narrative overview and assessment of the body of evidence derived from the comprehensive review of the studies. The reported outcomes will be summarised by study design, duration, intensity/frequency of intervention delivery and by the six-priority health promotion action areas set out in the Ottawa Charter. We will conduct a thematic analysis to identify the focus areas of current interventions. This systematic review protocol has been prepared according to the Preferred Reporting Items for Systematic reviews and Meta- analyses for Protocols 2015 statement.

Ethics and dissemination This study does not require ethics approval. We will disseminate the results of this review through peer-reviewed publications and conference presentations.

Trial registration number CRD42018110853.

\section{INTRODUCTION}

\section{Rationale}

In 2016, an estimated 41 million deaths globally ( $71 \%$ of all deaths) were due to
Strengths and limitations of this study

- This will be a comprehensive review that examines multiple workplaces, across various industries in low-income and middle-income countries.

- The review search dates and search strategy will ensure a comprehensive search for relevant articles.

- The methods of this review have been outlined in a protocol to guard against arbitrary decision making in the review process.

- Our search strategy is restricted by language; studies included will be limited to those in English.

- The inclusion of studies with diverse study designs, intervention types and workplace settings makes this a broad, heterogeneous study; this may limit the depth of the analysis.

non-communicable diseases (NCDs). ${ }^{1}$ The majority of these deaths were caused by: cardiovascular disease $(44 \%)$; cancer $(22 \%)$; chronic respiratory disease $(9 \%)$ and diabetes $(4 \%) .{ }^{1}$ In low-income and middle- income countries (LMICs), $85 \%$ of premature deaths are attributable to NCDs. ${ }^{2}$ In 2014, WHO estimated a $17 \%$ increase of deaths from NCDs globally and a $27 \%$ increase for the African region, equivalent to 28 million additional deaths by $2030 .{ }^{3}$ In sub-Saharan Africa region, where majority of the LMICs are located, $\mathrm{WHO}^{4}$ estimated that by 2020 , NCDs will be as prevalent as communicable diseases. Already, NCDs are the main cause of adult deaths in Mauritius, Namibia and Seychelles. ${ }^{3}$

The leading risks factors associated with the global increase in mortality are high blood pressure (responsible for $13 \%$ of death globally), tobacco use (9\%), high blood glucose $(6 \%)$, physical inactivity $(6 \%)$ and overweight and obesity (5\%). ${ }^{5}$ Changes in lifestyle, adoption of sedentary behaviours and nutrition transition have been identified as 
some of the modifiable risk factors that increase the risk of NCDs. ${ }^{2}$ The reversal or mitigation of this trend calls for the application of effective principles and practices of health promotion ${ }^{6}$ and the mainstreaming of health promotion. ${ }^{7}$ The current WHO strategy towards the prevention of NCDs incorporates the reduction of health risks and promotion of healthy lifestyles through health promotion. ${ }^{8}$

Health promotion is described as the process that enables people to increase control over (health determinants), and to improve their health'. ${ }^{9}$ The 1997 Jakarta declaration affirmed that health promotion strategies were indeed effective in addressing health risk factors, ${ }^{10}$ particularly lifestyle-related risk factors which can be modified to prevent disease. ${ }^{4}$

Globally, the health promotion approach has been adopted by many countries including the LMICs. For instance, Nyamwaya ${ }^{11}$ points out that the use of health promotion as a means of increasing societal responsibility for health now exists in all African countries. Laws and policies that facilitate adoption of healthy lifestyles and disease prevention such as tobacco legislation, have been put in place. ${ }^{11}$ A focus on settings for health promotion has enabled the creation of supportive environments through the development of relevant, practical health promotion interventions that address a full range of health determinants at each setting. ${ }^{12}$ The introduction of the settings approach for health promotion followed the 1986 Ottawa Charter's declaration that 'health is created and lived by people within the settings of their everyday life, where they learn, work, play and love'. ${ }^{9}$ The settings approach has translated to the utilisation of 'the health potentials inherent in the social and institutional settings of everyday life'. ${ }^{13}$ Settings identified in the Ottawa Charter included: prisons, schools, universities, market places, hospitals, islands, districts, cities, regions and workplaces. ${ }^{9}$

The workplace as a health promotion setting presents an opportunity to reach many people within the adult population. ${ }^{14}$ The working population is one that would not normally be engaged in organised health improvement initiatives. ${ }^{15}$ WHO has estimated that workers are estimated to represent half of the world's population ${ }^{16}$ and majority of them spend a substantial portion of their waking hours at work. ${ }^{17}$ Workplace health promotion (WHP) interventions are defined as employer initiatives directed at protecting the health of employees and thereby improving their productivity. ${ }^{18}$ The Centers for Disease Control and Prevention ${ }^{19}$ has described the three components to comprehensive WHP programmes as: screening, lifestyle or risk factor management and disease management. Workplaces may implement programmes that include one component or a combination of components. Examples of screening programmes include blood pressure and body weight measurement, and blood cholesterol level assessment. ${ }^{20}$ The majority of the WHP programmes target lifestyle or risk factor management at the individual level. Examples of these include: physical activity and nutrition programmes,${ }^{21} 22$ reduction in smoking ${ }^{2324}$ and use of stairs. ${ }^{25}$

WHP contributes to improvement of employee health and can help contain the current epidemic of lifestyle-related diseases. ${ }^{26}$ When properly designed and implemented, WHP interventions have been associated with multiple benefits. For instance, in a systematic review of literature carried out by Cancelliere et $a l^{27}$ the results from $21 \%$ of the studies show preliminary evidence that WHP programmes can positively affect presenteeism. Authors of a review that looked at WHP interventions for smoking cessation tested in controlled studies conclude that they found strong evidence that interventions which target individual smokers increase the likelihood of quitting smoking. ${ }^{28}$ A prospective cohort study that aimed to evaluate the impact of a 6-year WHP programme reported a decrease in systolic blood pressure in the hypertension subgroup. ${ }^{29}$ In an evaluation of a WHP programme, Oberlinner et $a t^{20}$ demonstrate that the programme yielded benefits in reduction of employee's body mass index. Results from a cluster randomised controlled trial investigating effectiveness of a WHP intervention showed that there were positive changes in job performance and psychological health of the employees. ${ }^{30}$

It is notable that reviews for health promotion interventions have limited their focus to individual-level interventions, leaving out interventions that focus on environmental, structural and social determinants of health. ${ }^{31}{ }^{32}$ With limited research on interventions focusing on multiple health determinants, employers have also shown reluctance to offer sufficiently comprehensive WHP programmes because they are not fully persuaded of their benefits, and they also contend that there are few best practices for them to emulate. ${ }^{1533}$ Moreover, most of the published research in WHP has been reported from high-income countries ${ }^{33}$ and there is scarcity of WHP reported in the LMICs. ${ }^{29}$ There is a gap in the provision of evidence-based health promotion interventions at the workplace. This review will yield a narrative overview and assessment of the body of evidence. The results of this review will provide additional information to guide strategic WHP choices and help identify 'best buy interventions'. Sustainability of WHP programmes refers to the continuation of interventions or the effects. ${ }^{34}$ Some studies have sighted employee participation rates as an example for indication of sustainability of the WHP interventions. ${ }^{35} 36$ There is limited information on the long-term effectiveness and continuation of the WHP programmes. ${ }^{34} 36$

Overall, a systematic review that synthesises multiple published studies on WHP from LMICs will provide a comprehensive summary of evidence available in WHP practice in these countries. Like the publication of primary research studies mentioned earlier, most of the literature reviews carried out on WHP also focus on studies done in high-income countries. ${ }^{37-39}$ Results from this review will provide preliminary evidence for WHP effectiveness and sustainability specific to LMICs. Such 
evidence will facilitate the scaling up of the implementation of effective, feasible interventions within LMICs. We therefore propose to carry out a systematic review that aims to synthesise published studies on current WHP practice in LMICs countries focusing on effectiveness and sustainability of the interventions.

\section{Objective}

To assess the effectiveness and sustainability of interventions for health promotion in the workplace setting in LMICs.

We aim to address the following questions:

1. How effective are interventions for health promotion at the workplace setting in LMICs?

2. How sustainable are interventions for health promotion at the workplace setting in LMICs?

\section{METHODS}

This review protocol is registered in the PROSPERO International prospective register of systematic reviews (registration number: CRD42018110853). The review will be prepared according to the Preferred Reporting Items for Systematic reviews and Meta-Analysis Protocols (PRISMA-P) 2015 statement. $^{40}$

\section{Inclusion criteria}

a) Population: this review will include studies done in adult populations; 18 years of age and above, within the workplace settings in LMICs.

b) Intervention characteristics: interventions for health promotion at the workplace setting.

Study designs: all study designs will be included since WHP interventions are evaluated using a wide variety of approaches and study designs. ${ }^{41-43}$

Publication status: published studies whose full text is publicly available. We will review the reference lists from past reviews for suitable studies that would meet the inclusion criteria. Duplicate publications of the same material will be excluded. For studies published in multiple papers, the most recent version will be considered.

Timeline: studies published from LMICs up to 31 March 2019.

Language: English.

c) Comparison: studies on WHP will present multiple research designs. An intervention to promote health at the workplace will be compared with no intervention. To help answer our research questions, other comparisons involving interventions will be assessed on a case-to-case basis as encountered in the literature.

d) Outcomes measured either objectively or subjectively will be included in the review. This will include primary outcomes; employee participation rates, duration of intervention, objectively or subjectively measured effects of the intervention on employee's physical, mental, financial or social health measures. As a secondary outcome, operational indicators and factors for sustainability will be considered.

\section{Box 1 Search strategy}

\section{Search terms}

"Workplace" OR "occupational" OR "worksite" OR "organi*ational" OR "industrial" OR "work" OR "worker" OR "employee"

AND

"Health*” OR "health promotion" OR "Wellness" OR "Well-being" "wellbeing" OR "health management" OR " Health protection"

AND

"Program*" OR "framework" OR "model" OR "intervention" $O R$ "initiative"

AND

"Afghanistan" OR "Albania" OR "Algeria" OR "American Samoa" OR "Angola" OR "Armenia" OR "Azerbaijan" OR "Bangladesh" OR "Belarus" OR "Belize" OR "Benin" OR" "Bhutan" OR "Bolivia" OR "Bosnia and Herzegovina" OR "Botswana" OR "Brazil" OR "Bulgaria" OR "Burkina Faso" OR "Burundi" OR "Cabo Verde" OR "Cambodia" OR "Cameroon" OR "Central African Republic" OR "Chad" OR "China" OR "Colombia" OR "Comoros" OR "Democratic Republic of Congo" OR "Congo" OR "Costa Rica" OR "Cote d'Ivoire" OR "Ivory Coast" OR "Cuba" OR "Djibouti" OR "Dominica" OR "Dominican Republic" OR "Ecuador" OR "Egypt" OR "Arab Republic" OR "El Savador" OR "Equatorial Guinea" OR "Eritrea" OR "Eswatini" OR "Ethiopia" OR "Fiji" OR "Gabon" OR "The Gambia" OR "Georgia" OR "Ghana" OR "Grenada" OR "Guatamela" OR "Guinea" OR "Guinea Bissau" OR "Guyana" OR "Haiti" OR "Honduras" OR "India" OR "Indonesia" OR "Iran" OR "Islamic Republic" OR "Iraq" OR "Jamaica" OR "Jordan" OR "Kazakhastan" OR "Kenya" OR "Kiribati" OR "Democratic People's Republic of Korea" OR "Korea" OR "Kosovo" OR "Kyrgyz Republic" OR "Lao PDR" OR "Lebanon" OR "Lesotho" OR "Liberia" OR "Libya" OR "Madagascar" OR "Malawi" OR "Malaysia" OR "Maldives" OR "Mali" OR "Marshall Islands" OR "Mauritania" OR "Mauritius" OR "Mexico" OR "Micronesia" OR "Moldova" OR "Mongolia" OR "Montenegro" OR "Morocco" OR "Mozambique" OR "Myanmar" OR "Namibia" OR "Nauru" OR "Nepal" OR "Nicaragua" OR "Niger" OR "Nigeria" OR "North Macedonia" OR "Pakistan" OR "Papua New Guinea" OR "Paraguay" OR "Peru" OR "Philippines" OR "Romania" OR "Russian Federation" OR "Rwanda" OR "Samoa" OR "Sao Tome and Principe" OR "Senegal" OR "Serbia" OR "Sierra Leonne" OR "Solomon Islands" OR "Somalia" OR "South Africa" OR "South Sudan" OR "Sri Lanka" OR "St Lucia" OR "St Vincent and the Grenadines" OR "Sudan" OR "Suriname" OR "Syrian Arab Republic" OR "Tajikistan" OR "Tanzania" OR "Thailand" OR "Timor-Leste" OR "Togo" OR "Tonga" OR "Tunisia" OR "Turkey" OR "Turkmenistan" OR "Tuvalu" OR "Uganda" OR "Ukraine" OR "Uzbekistan" OR "Vanuatu" OR "Venezuela" OR "Vietnam" OR "West Bank of Gaza" OR "Yemen" OR "Zambia" OR "Zimbabwe" OR Africa OR "sub-Saharan Africa" OR "low and middle income countr" OR "low income countr" OR "Low OR middle income countr" OR "Low and middle income countr*" OR "LMIC*" OR "developing country" OR "underdeveloped country" OR "resource limited"

\section{Exclusion criteria}

Workplace-related studies that do not report on WHP interventions will be excluded, for example, studies reporting on a standalone aspect such as occupational safety and health, hospitals or treatment.

\section{Data sources and search strategy}

We will search the following databases: EMBASE, MEDLINE, PubMed, Web of Science, Scopus, ProQuest, CINAHL. 
Box 1 shows the search strategy that we will use. We will adapt the strategy to the different databases.

Grey literature

To allow for the inclusion of as much evidence as possible, we will use Google web search (www.google.com) to look for grey literature. We will contact the first and senior author of included articles for relevant material. We will do this through email communication.

\section{Study records}

Data management

We will import all identified studies to EndNote software where duplicate records will be identified and excluded from record. In our study selection process, we will be guided by the inclusion criteria. We will use Rayyan QCRI ${ }^{44}$ an internet-based program to assist the screening and selection of studies.

\section{Screening}

Two reviewers (MNW and BZ-D) will independently select all studies that meet the inclusion criteria. The reviewers will screen the titles and abstracts of the studies for relevance based on the criteria set. They will then screen the full texts of potential eligible studies for inclusion and relevance. Any disagreements will be resolved by consensus. The details of the excluded studies outlining reasons for exclusion will be documented and presented in a flow chart.

\section{Data extraction}

Using a predetermined data extraction sheet, two reviewers will independently extract data from final full texts of eligible studies and any inconsistencies will be resolved by consensus.

\section{Data items}

We will extract the following data from our final selection: details of publication (author, author country of affiliation, year of publication, title of article, name of journal study published in), geographical location of intervention, study context (workplace/industrytype, single or multiple organisations studied), subjects of research (role/description of target population, if study is gender specific, employment type of participants, profession), aim of the study, programme/intervention priority area focus, sustainability aspects of programme, methods, study outcomes, study conclusions, limitations and future research areas proposed.

\section{Risk of bias and quality appraisal}

Two reviewers will independently rate the quality and risk of bias in included studies using two tools from Cochrane handbook for systematic reviews of interventions. To assess the quality of studies included, the reviewers will use the criteria from Cochrane handbook for systematic reviews on international version 5.1.0. ${ }^{45}$ To assess the risk of bias, the reviewers will use the Cochrane tool (table 1) commonly used for random controlled trials. This will be adapted to this review to accommodate the multiple research designs anticipated in the included studies. The adaptation will be done as per the guidelines and criteria for judging risk of bias in the 'risk of bias' assessment tool. ${ }^{46}$ We will assign a judgement of 'low', 'high' or 'unclear risk' of bias in the review authors' judgement

Table 1 The Cochrane tool for assessing risk of bias

Domain

\section{Selection bias}

Random sequence generation: selection bias (biased allocation to interventions) due to inadequate generation of a randomised sequence.

Allocation concealment: selection bias (biased allocation to interventions) due to inadequate concealment of allocations prior to assignment.

\section{Performance bias}

Blinding or participants and personnel: performance bias due to knowledge of the allocated interventions by participants and personnel during the study.

Blinding of outcome assessment: detection bias due to knowledge of the allocated interventions by outcome assessors.

\section{Attrition bias}

Incomplete outcome data: attrition bias due to amount, nature or handling of incomplete outcome data.

\section{Reporting bias}

Selective reporting: reporting bias due to selective outcome reporting.

\section{Other bias}

Bias due to problems not covered elsewhere in the table. 
column. Additional categories indicating either uncertainty or lack of information over the potential for bias will be incorporated.

For all non-randomised studies, we will incorporate an assessment of risk of bias due to confounders. We will compile a list of confounders and determine which of these confounders were considered in the selected studies. The assessment will include determining if the most important confounders were considered, how precisely each confounder was measured, whether they were distributed similarly in intervention and control cohorts, how carefully they were controlled for and how the researchers controlled for confounding. ${ }^{45}$

Towards the detection of reporting bias, the authors will use funnel plots to demonstrate the intervention estimates from individual studies against a measure of each study's size.

\section{Data synthesis}

We will present a narrative overview and assessment of the body of evidence derived from the comprehensive review of the included studies. The studies will be presented and described by geographical region, regional spread of study authors, number of studies per year, journals that have published these studies. Additional characteristics of included studies will include: study design, duration of study, type of workplaces setting and description of research participants and intervention, study outcomes and any additional notes by the authors.

The summary assessment of risk of bias will be considered for each important outcome within each study (across domains) and across studies presented in summary tables. We will use the summaries to make judgements about the quality of evidence. We will create additional tables listing the identified confounders as columns and the studies as rows, indicating the results of assessments of each confounder for every study. We will also develop a table of comparisons and outcomes. A comparison of results will be done between results from studies assessed at high or unclear risk of bias and from those studies at low risk of bias. Comparison will further be drawn between outcomes for the various study designs, durations of delivery for each study, frequency of intervention delivery reported and priority health promotion action area that each WHP programme focuses on. The six health promotion priority areas outlined in the Ottawa Charter ${ }^{9}$ will be applied.

Assessment for sustainability will be adopted from the conceptual frameworks developed by Shediac-Rizkallah and Bone ${ }^{47}$ and adopted by Cochrane handbook for systematic reviews in public health and health promotion. The operational indicators that will be assessed will be categorised as follows: maintenance of health benefits achieved through an initial programme, level of institutionalisation of a programme within an organisation and measures of capacity building in the workplace setting. To evaluate specific conditions or strategies that favour sustainability in the LMICs context, a criterion assessing three groups of factors will be applied: project design and implementation factors, factors within the organisational setting and factors in the broader community environment such as cultural factors. ${ }^{47}$

Additionally, the reviewers will carry out a thematic analysis to present and discuss the main themes across different workplace types, time periods and geographical distribution of included studies. In addition to the manual data entry and summary, we will complement this analysis with the use of Leximancer V.4 software.

\section{Patient and public involvement}

We will not involve patients and the public in this review.

\section{Reporting this review}

We will report the systematic review according to the checklist of items to include when reporting a systematic review as per the PRISMA 2009 statement. ${ }^{48}$ We will present a flow diagram to show the study selection process, specifying reasons for exclusion at each stage. The study quality appraisal tool will be availed as online supplementary material.

\section{Potential amendments}

In case of any changes to this protocol, we will outline the details of the changes in the final report. However, no further amendments to this protocol are foreseen.

\section{CONCLUSION}

To heed to the WHO's clarion call to implement and scale-up effective health promotion interventions in Africa, ${ }^{3}$ there is a need to assess what has been effective and sustainable in the context of the workplace setting. There is need to '...establish what has worked.... and what should be done here and now, to improve the health of the people in Africa' ${ }^{3}$ and the rest of the LMICs.

Previous reviews on WHP focused on the effectiveness of specific interventions, for example, on physical activity, ${ }^{49} 50$ nutrition promotion ${ }^{51}$ and smoking cessation. ${ }^{28}$ Through this comprehensive review, we will provide new insights by presenting a holistic outline of current WHP practice in LMICs, with a focus on effectiveness and sustainability.

\section{Ethics and dissemination}

Since systematic reviews are based on available published data, this review will therefore not require any formal ethical approval. We will disseminate the results of this systematic review through peer-reviewed publications and conference presentations.

Contributors MNW conceived the paper and wrote the first draft. BZ-D and JLV provided revisions to the manuscript. All authors read and approved the final manuscript. MNW is the guarantor of the review.

Funding The authors have not declared a specific grant for this research from any funding agency in the public, commercial or not-for-profit sectors.

Competing interests None declared.

Patient consent for publication Not required.

Provenance and peer review Not commissioned; externally peer reviewed. 
Open access This is an open access article distributed in accordance with the Creative Commons Attribution Non Commercial (CC BY-NC 4.0) license, which permits others to distribute, remix, adapt, build upon this work non-commercially, and license their derivative works on different terms, provided the original work is properly cited, appropriate credit is given, any changes made indicated, and the use is non-commercial. See: http://creativecommons.org/licenses/by-nc/4.0/.

\section{REFERENCES}

1. World Health Organisation. World Health Statistics. 2018 https:// apps.who.int/iris/bitstream/handle/10665/272596/9789241565585eng.pdf?ua=1 (26 Nov 2018).

2. WHO. Non communicable diseases. 2019 https://www.who.int/en/ news-room/fact-sheets/detail/noncommunicable-diseases (20 Mar 2019).

3. WHO. The African Regional Health Report: The Health of the People. 2014 www.who.int/bulletin/africanhealth/en.

4. WHO. Chronic diseases and health promotion: Preventing chronic diseases: a vital investment. 2005 http://www.who.int/chp/chronic_ disease_report/contents/foreword.pdf?ua $=1$.

5. WHO. Health Promotion: 7th Global Conference on Health Promotion, Nairobi Call to Action. 2009a http://www.who.int/ healthpromotion/conferences/7gchp/en/.

6. Sampson KA, Amuyunzu-Nyamongo UM, Mensah G. AmuyunzuNyamongo, and G. Mensah, Health Promotion and Cardiovascular Disease Prevention in Sub-Saharan Africa. : WHO. Global Health Risks. 2009b, 2013:56: 344-55. http://www.who.int/healthinfo/ global_burden_disease/GlobalHealthRisks_report_full.pdf..

7. WHO. Global Health Risks. 2009b www.who.int/healthinfo/global_ burden_disease/GlobalHealthRisks_report_full.pdf.

8. WHO. Noncommunicable diseases and their risk factors. Prevention of noncommunicable diseases. 2019 https://www.who.int/ncds/ prevention/introduction/en/ (25 Mar 2019).

9. WHO. The Ottawa Charter for Health promotion: an International Conference on Health Promotion, the move towards a new public health. 1986 www.who.int/healthpromotion/conferences/previous/ ottawa/en/ (21 Nov 2019).

10. WHO. Health Promotion: Jakarta Declaration on Leading Health Promotion into the 21st Century. 1997 http://www.who.int/ healthpromotion/conferences/previous/jakarta/declaration/en/.

11. Nyamwaya D. Health promotion in Africa: strategies, players, challenges and prospects. Health Promot Int 2003;18:85-7.

12. Nutbeam D. What would the Ottawa Charter look like if it were written today? Crit Public Health 2008;18:435-41.

13. Kickbusch and Ilona, Tribute to Aaron Antonovsky - 'What creates health'. Health Promotion International. 1996;11:5-6.

14. Lankford T, Lang J, Bowden B, et al. Workplace health: engaging business leaders to combat obesity. J Law Med Ethics 2013;41 Suppl 2:40-5.

15. Goetzel RZ, Ozminkowski RJ. The health and cost benefits of work site health-promotionprograms. Annu Rev Public Health 2008;29:303-23.

16. WHO. Workers' health: global plan of action. $2007 \mathrm{http}: / /$ www.who. int/occupational_health/WHO_health_assembly_en_web.pdf?ua=1.

17. Conrad P. Health and fitness at work: a participants' perspective. Soc Sci Med 1988;26:545-50.

18. Harden A, Peersman G, Oliver S, et al. A systematic review of the effectiveness of health promotion interventions in the workplace. Occup Med 1999;49:540-8.

19. CDC. Workplace Health Promotion. 2019 https://www.cdc.gov/work placehealthpromotion/index.html. [30 Mar 2019].

20. Oberlinner C, Lang S, Germann C, et al. [Prevention of overweight and obesity in the workplace. BASF-health promotion campaign "trim down the pounds-losing weight without losing your mind"]. Gesundheitswesen 2007;69:385-92.

21. Robroek SJ, van Lenthe FJ, Burdorf A. The role of lifestyle, health, and work in educational inequalities in sick leave and productivity loss at work. Int Arch Occup Environ Health 2013;86:619-27.

22. Chalupka S. Workplace obesity prevention. Aaohn J 2011;59:236.

23. Terry PE, Seaverson EL, Staufacker MJ, et al. The effectiveness of a telephone-based tobacco cessation program offered as part of a worksite health promotion program. Popul Health Manag 2011;14:117-25.

24. O'Connell KA, Hosein VL, Schwartz JE. Thinking and/or doing as strategies for resisting smoking. Res Nurs Health 2006;29:533-42.

25. Eves FF, Webb OJ, Griffin C, et al. A multi-component stair climbing promotional campaign targeting calorific expenditure for worksites; a quasi-experimental study testing effects on behaviour, attitude and intention. BMC Public Health 2012;12:423.

26. Mattke S, Liu H, Caloyeras J, et al. Workplace Wellness Programs Study: Final Report. Rand Health Q 2013;3:7.

27. Cancelliere C, Cassidy JD, Ammendolia C, et al. Are workplace health promotion programs effective at improving presenteeism in workers? A systematic review and best evidence synthesis of the literature. BMC Public Health 2011;11:395.

28. Moher M, Hey K, Lancaster T, et al. Workplace interventions for smoking cessation. Cochrane Database Syst Rev 2005:CD003440.

29. Eng JY, Moy FM, Bulgiba A. Impact of a Workplace Health Promotion Program on Employees' Blood Pressure in a Public University. PLoS One 2016;11:e0148307.

30. Edwardson CL, Yates T, Biddle SJH, et al. Effectiveness of the Stand More AT (SMArT) Work intervention: cluster randomised controlled trial. BMJ 2018;363:363.

31. Wilson MG, Holman PB, Hammock A. A comprehensive review of the effects of worksite health promotion on health-related outcomes. Am $J$ Health Promot 1996;10:429-35.

32. Jackson N, Waters E. Guidelines for Systematic Reviews in Health Promotion and Public Health Taskforce. Criteria for the systematic review of health promotion and public health interventions. Health Promot Int 2005;20:367-74.

33. Linnan L, Bowling M, Childress J, et al. Results of the 2004 National Worksite Health Promotion Survey. Am J Public Health 2008;98:1503-9.

34. Swerissen H, Crisp BR. The sustainability of health promotion interventions for different levels of social organization. Health Promot Int 2004;19:123-30.

35. Robroek SJ, van Lenthe FJ, van Empelen P, et al. Determinants of participation in worksite health promotion programmes: a systematic review. Int J Behav Nutr Phys Act 2009;6:26.

36. Lowensteyn I, Berberian V, Berger C, et al. The Sustainability of a Workplace Wellness Program That Incorporates Gamification Principles: Participant Engagement and Health Benefits After 2 Years. Am J Health Promot 2019:089011711882316.

37. Malik SH, Blake H, Suggs LS. A systematic review of workplace health promotion interventions for increasing physical activity. $\mathrm{Br} \mathrm{J}$ Health Psychol 2014;19:149-80.

38. Wolfenden L, Goldman S, Stacey FG, et al. Strategies to improve the implementation of workplace-based policies or practices targeting tobacco, alcohol, diet, physical activity and obesity. Cochrane Database Syst Rev 2018;11:CD012439.

39. Freak-Poli RL, Cumpston M, Peeters A, et al. Workplace pedometer interventions for increasing physical activity. Cochrane Database Syst Rev 2013;4:CD009209.

40. David Moher LS, Clarke M, Ghersi D, et al. Stewart and PRISMA-P Group Preferred reporting items for systematic review and metaanalysis protocols (PRISMA-P) 2015 statement, 2015.

41. Baum F. Researching public health: behind the qualitativequantitative methodological debate. Soc Sci Med 1995;40:459-68.

42. Donner A, Klar N. Pitfalls of and controversies in cluster randomization trials. Am J Public Health 2004;94:416-22.

43. Glasziou P, Vandenbroucke JP, Chalmers I. Assessing the quality of research. BMJ 2004;328:39-41.

44. Mourad Ouzzani HH, Fedorowicz Z, Elmagarmid A. Rayyan - a web and mobile app for systematic reviews. , 2016:5, 210. [cited 201815 Sep 2018].

45. Reeves BC, et al. Including non-randomized studies, in Cochrane Handbook for Systematic Reviews of Interventions, Higgins JPT GS, eds. 2011. The Cochrane Collaboration.

46. Higgins A. Sterne JAC Assessing risk of bias in included studies, 2011. Cochrane Handbook for Systematic Reviews of Interventions.

47. Shediac-Rizkallah MC, Bone LR. Planning for the sustainability of community-based health programs: conceptual frameworks and future directions for research, practice and policy. Health Educ Res 1998;13:87-108.

48. Moher D, Liberati A, Tetzlaff J, et al. Preferred reporting items for systematic reviews and meta-analyses: the PRISMA statement. PLOS Med 2009;6:e1000097.

49. Marshall AL. Challenges and opportunities for promoting physical activity in the workplace. J Sci Med Sport 2004;7(1 Suppl):60-6.

50. Proper KI, Koning M, van der Beek AJ, et al. The effectiveness of worksite physical activity programs on physical activity, physical fitness, and health. Clin J Sport Med 2003;13:106-17.

51. Janer G, Sala M, Kogevinas M. Health promotion trials at worksites and risk factors for cancer. Scand J Work Environ Health 2002;28:141-57. 